\title{
Dephasing of a quantum dot due to the Coulomb interaction with a gate electrode
}

\author{
S. R. Woodford* and A. Bringer \\ Institut für Festkörperforschung (IFF), Forschungszentrum Jülich, D-52425, Germany and \\ Center of Nanoelectronic Systems for Information Technology (CNI), Forschungszentrum Jülich, D-52425, Germany \\ K. M. Indlekofer \\ Institute of Bio- and Nanosystems (IBN-1), Center of Nanoelectronic Systems for Information Technology (CNI), \\ Forschungszentrum Jülich, D-52425 Jülich, Germany
}

\begin{abstract}
Dephasing of quantum dots presents an intrinsic limitation on their usage for quantum computing. We consider the dephasing of a double quantum dot caused by the Coulomb interaction with the nearby gate electrodes, and show that this occurs on a much longer timescale than dephasing due to phonons. However, the effect grows rapidly stronger as the system size decreases, and therefore it is expected to limit potential miniaturization of these systems.
\end{abstract}

PACS numbers: 03.65.Yz, 73.21.La, 85.35.Be

\section{INTRODUCTION}

The development of a functional quantum computer is an important goal that has attracted much attention in recent years. The importance of quantum computing is due to the existence of "quantum algorithms" which solve certain problems (e.g. prime factorization [1]) exponentially faster than they can be solved on a classical computer. This speedup is possible because the algorithms exploit the quantum mechanical properties (such as superposition and entanglement) of the quantum bits (qubits) [2, 3].

However, since quantum mechanical systems can never be isolated completely from their environment, a prepared quantum state will decay with time. This passage from a quantum into a classical state involves two different types of decay — dephasing and dissipation. Dephasing refers to the loss of phase coherence of the superposition of states, i.e. the decay of the off-diagonal elements of the density matrix, and dissipation describes the flow of energy out of the system, characterized by the decay of the diagonal elements of the density matrix. Both mechanisms destroy the quantum mechanical nature of the system. A system may therefore only be a viable option for a quantum computer if its dephasing and dissipation times are much longer than the average computation time $[2,3]$.

Solid state systems have emerged as promising candidates for quantum computing, due to their scalability and the wealth of expertise available in fabrication and measurement technology. Proposals for solid state quantum computers are usually either based on superconductivity, in which a few macroscopic variables act as the quantum states $[4-6]$, or semiconductor systems such as quantum dots $[7,8]$ or periodic dopant arrays $[9,10]$, in which individual electrons carry the information. In this paper,

*Electronic address: s. woodford@fz-juelich.de we consider a charge qubit, which consists of a pair of quantum dots with a single electron tunneling between them.

Two distinct types of dephasing occur in such a system. The first occurs as the initial (experimentally prepared) state evolves into a quasiparticle state of the many-body system. For example, a bare exciton excited by a photon will rapidly lose coherence when "dressed" with phonons [11]. Similarly, an electron beam passing through a quantum dot dephases as it induces an image charge in a nearby point contact $[12-16]$. We shall refer to this type of dephasing as "initialization dephasing". It is caused by renormalization and, if the environment (i.e. everything apart from the quantum dot system) is stationary, it has only limited effect - it ceases to act once a quasiparticle state is formed. (If the environment is nonstationary, as in a current-based "which-path" detector [12-16], a true quasiparticle state cannot form; initialization dephasing may be unlimited in this case.).

We focus here on the second type of dephasing, which is caused by interactions of the quasiparticle state with the environment. This proceeds either via scattering of the quasiparticle, or by the interaction of the quasiparticle with fluctuations (both quantum and thermal). We refer to this as "dynamical dephasing". Typically, dynamical dephasing acts on a much slower timescale than initialization dephasing, but is unlimited in effect; it will eventually lead to complete decoherence of the system. For example, a bare exciton will be dressed by LA phonons within several picoseconds of excitation, causing up to $25 \%$ loss of coherence [11]. The dressed exciton is more stable, however, and subsequent dephasing occurs on the timescale of nanoseconds [17, 18].

Theoretical investigations of dynamical dephasing usually focus on electron-phonon scattering [17, 18]. In this paper we consider an alternative dephasing mechanism - the interaction of the electron with nearby gate electrodes. Gate electrodes are metallic strips used to define and control the potential surface of the quantum dots and to prepare initial states (e.g. the "left" or "right" 
charge states [18]). Although they are typically assumed to contribute only a classical field, this is not necessarily true [19]. Dephasing due to gate electrodes has been considered in Ref. [10], where the electrodes are treated as a noninteracting boson gas, coupled to the quantum dot by the "spin-boson" interaction. Instead of using this highly-idealized coupling mechanism, we use the more realistic Coulomb interaction. We also do not make the Markov approximation; memory effects in the gate are included using the kinetic equation approach described in Ref. [20]. (The term "kinetic equation" is taken from the work of Loss and Schoeller [21]; in Ref. [20], the same equation is referred to as a "generalized master equation".)

Unlike the Coulomb-induced dephasing present in "which-path" detectors [12-16], our Coulomb bath (comprising the gate electrodes) has zero current. We therefore neglect the initialization dephasing and assume that the image charge in the gate electrodes, and all related renormalization effects, are included in the initial conditions.

In Section II we present our model in detail and describe our basic assumptions. In Section III we present the kinetic equation that describes the evolution of the quantum dot density matrix. This equation contains the retarded density-density correlation function of the gate electrode, which is shown in Section III to be related to the generalized reflectivity coefficient of the metal. (Using the Drude approximation [22], this may be calculated explicitly.) We then demonstrate how the Laplace transform aids in the solution of the kinetic equation. In Section IV we present our numerical results of the dissipation and dephasing times of a GaAs charge qubit, as functions of various parameters. Our conclusions are presented in Section V. The detailed derivation of the kinetic equation is contained in Appendix A, while a description of our numerical methods is presented in Appendix B. Unless otherwise stated, Hartree atomic units are used throughout.

\section{DESCRIPTION OF THE PROBLEM}

A schematic diagram of a typical charge qubit is shown in Fig. 1(a) (see e.g. Refs. [18, 23]). A 2-dimensional electron gas is formed at the interface between GaAs and AlGaAs. A double quantum dot structure [indicated by the dashed lines in Fig. 1(a)] is then defined by charging the gate electrodes to different potentials. In a typical experiment (see e.g. Refs. $[10,18]$ ), the system is initialized by setting the potential of one of the dots much lower than the other, so that the electron rests within that dot. At $t=0$, the potentials are nonadiabatically equalized, and tunneling begins. After a time, the potentials are shifted again and a measurement is made. Dephasing can be estimated from the difference between the result of the measurement and the expected result (calculated from elementary quantum mechanics). We study only the de- phasing part of this process; that is, we fix the initial state and keep the potential constant throughout.

Before we can study the dynamics of dephasing, several approximations are needed. First, we consider the gate electrodes. As shown in Fig. 1(a), there are several of these, all of which may be at different potentials. From the point of view of the quantum dot, each electrode has two separate effects: the potential of each electrode statically defines the two quantum dots, and each electrode exhibits a dynamical response to the motion of the electron. Since the potential of an electrode has a negligible effect on its Fermi level, plasmon frequency and electron mean free path, each electrode will have a similar dynamical response. From the dynamical point of view, there is little difference between a system with several separate electrodes and a system with a single, large electrode. (The primary differences are the increase of interaction area and the neglect of eddy currents, both of which are small effects in this system.) However, the correlation functions of the latter are far more amenable to calculation. We therefore separate the contribution from the gate electrodes into a static part, which defines the potential landscape of the quantum dots, and a dynamical part, which is taken to be an infinite metal layer. This is shown schematically in Fig. 1(b).

Next, we consider the quantum dot system. We assume that the confinement in the $z$ direction is significantly stronger than that in the $x$ or $y$ directions, so that the wavefunction may be written

$$
\varphi_{i}(\mathbf{r})=\sqrt{\delta(z)} \varphi_{i}\left(\mathbf{r}_{\|}\right)
$$

In the $x$ and $y$ directions, we assume that each quantum dot is a square-well potential with infinitely high walls except in the tunneling direction. The height of the tunnel barrier is chosen so that the tunneling energy matches that of experiment, and both wells have the same depth. We assume that only the lowest energy bonding and antibonding wavefunctions are relevant; these are constructed numerically (see Appendix B for details).

As mentioned previously, we assume that the renormalization due to the image charge is already taken into account in the form of the potential landscape of the quantum dots. Thus the static image charge of the simplified system [i.e. the single electrode system depicted in Fig. 1(b)] vanishes.

Finally, we assume that at $t=0$, there is no correlation between the gate and the electron in the qubit. This means that for $t<0$, the quantum dot system behaves classically, and at $t=0$ we "turn on" the quantum mechanics. This is equivalent to assuming that at $t=0$, we may prepare the dot very rapidly in a particular quantum state, thus breaking all correlations with the gate. 

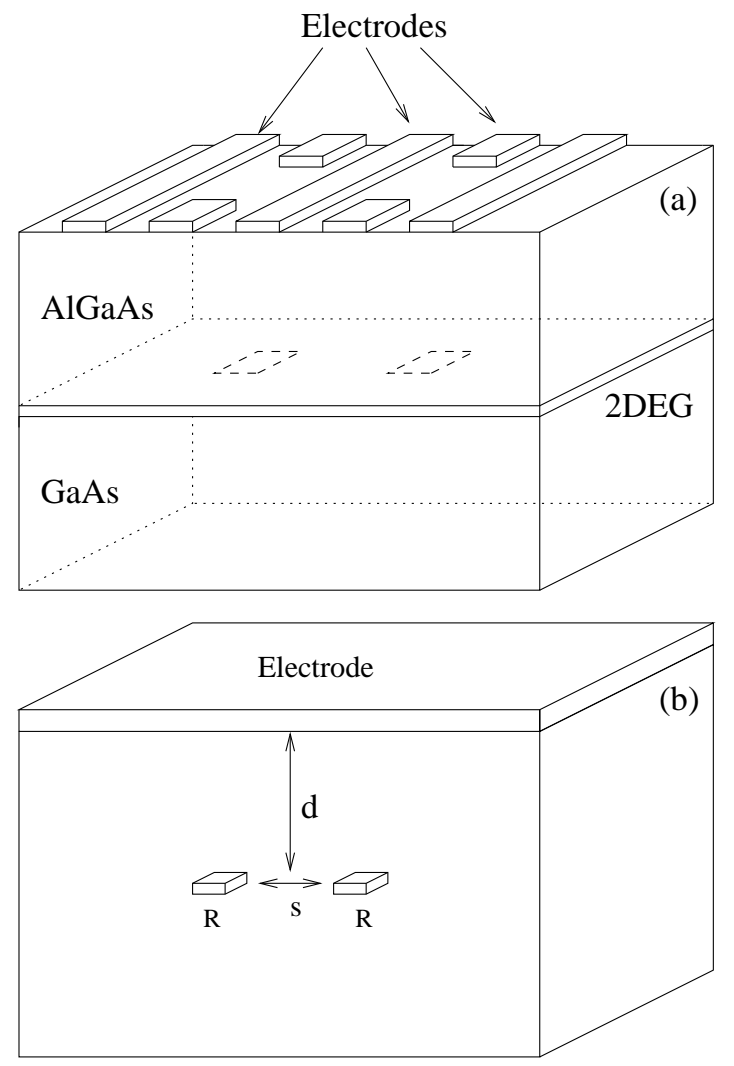

FIG. 1: (a) A schematic drawing of a double quantum dot system. The electrodes define the quantum dots (indicated by the dashed lines), control tunneling into the source or drain electrodes (not shown) and determine the tunnel barrier. (b) An approximated system, in which the electrodes are replaced by a single, infinite electrode, and the double quantum dot system is represented with a pair of square well potentials of equal height connected via a tunnel barrier. The gate electrode is assumed thick enough that it may be approximated as a bulk metal (filling the region $z>d$ ).

\section{EQUATIONS OF MOTION}

\section{A. The kinetic equation}

The closed quantum system, consisting of the gate electrode and the double quantum dot, may be described by the Hamiltonian

$$
\begin{aligned}
& H=\sum_{i=1}^{2} E_{i} a_{i}^{\dagger} a_{i}+H_{B} \\
& +\sum_{i, j} \int d \mathbf{r} d \mathbf{r}^{\prime} \frac{1}{\epsilon_{r}\left|\mathbf{r}-\mathbf{r}^{\prime}\right|} \varphi_{i}^{*}(\mathbf{r}) \varphi_{j}(\mathbf{r}) \Psi^{\dagger}\left(\mathbf{r}^{\prime}\right) \Psi\left(\mathbf{r}^{\prime}\right) a_{i}^{\dagger} a_{j} .(2)
\end{aligned}
$$

The double quantum dot is expressed in the energy eigenfunction basis (i.e. $\varphi_{1}$ and $\varphi_{2}$ are the bonding and antibonding orbitals, respectively), with $a_{i}^{\dagger}$ and $a_{i}$ respectively the creation and annihilation operators in this basis. The position representation is used for the gate electrons. $H_{B}$ is the Hamiltonian for the bare gate, and $\epsilon_{r}$ is the dielectric constant of the semiconductor medium between the dot and the gate.

The position representation is preferable for describing the gate, since it shows explicitly that the interaction couples the quantum dot states to the electron density of the gate [via the operator $\left.n\left(\mathbf{r}^{\prime}\right)=\Psi^{\dagger}\left(\mathbf{r}^{\prime}\right) \Psi\left(\mathbf{r}^{\prime}\right)\right]$. This has the advantage that the density-density correlation function, which we use below, takes the form of a 2-point correlator; in any other basis, it appears as a 4-point correlator (with two creation operators and two annihilation operators). However, Eq. (2) is not the spin-boson model, since the Hamiltonian $H_{B}$ contains fermionic terms that cannot be expressed in terms of the bosonic operator $n(\mathbf{r})$.

In deriving the equations of motion, we may choose the eigenfunctions of the quantum dot to be real-valued, which allows some simplification. Furthermore, the static image charge vanishes (as described in Section II):

$$
<n(\mathbf{r})>=\frac{\operatorname{Tr}\left[\exp \left(-\beta H_{B}\right) n(\mathbf{r})\right]}{\operatorname{Tr}\left[\exp \left(-\beta H_{B}\right)\right]}=0 .
$$

To describe the dynamics, we denote the density operator of the combined dot-gate system by $W(t)$. This operator contains all possible information concerning the evolution of the full system. To describe the dynamics of the quantum dot, we define the reduced density operator, $\rho(t)=\operatorname{Tr}_{B}[W(t)]$, where $\operatorname{Tr}_{B}$ traces over all gate variables. The operator $\rho(t)$ is the many-body density operator that describes the quantum dot; since we consider only the one particle sector of this operator, $\rho(t)$ is identical to the single-particle density operator. We may therefore express it as a density matrix $\rho_{i j}(t)$ relative to the energy eigenfunction basis spanned by $\left\{\varphi_{1}, \varphi_{2}\right\}$.

The evolution of $\rho_{i j}(t)$ is governed by a kinetic equation [20]. This equation is derived by projecting the dynamics of $W(t)$ onto the quantum dot subspace (for details, see Appendix A). The evolution of the gate system under the influence of the dot is taken into account by the kinetic equation, which leads to a retarded interaction of the dot with itself. The kinetic equation is exact, but contains all orders of the interaction Hamiltonian. To obtain more tractable equations, we truncate the kinetic equation at second order (second order Born approximation), after which the equations of motion for $\rho_{i j}(t)$ become 


$$
\begin{array}{r}
\dot{\rho}_{11}(t)=\int_{0}^{t}\left\{\rho_{22}\left(t-t^{\prime}\right)\left[e^{i E_{21} t^{\prime}} R_{1221}^{>}\left(t^{\prime}\right)+e^{-i E_{21} t^{\prime}} R_{1221}^{<}\left(t^{\prime}\right)\right]-\rho_{11}\left(t-t^{\prime}\right)\left[e^{i E_{21} t^{\prime}} R_{1221}^{<}\left(t^{\prime}\right)+e^{-i E_{21} t^{\prime}} R_{1221}^{>}\left(t^{\prime}\right)\right]\right. \\
\left.+\rho_{12}\left(t-t^{\prime}\right) e^{i E_{21} t^{\prime}}\left[R_{1112}^{<}\left(t^{\prime}\right)-R_{1222}^{>}\left(t^{\prime}\right)\right]+\rho_{21}\left(t-t^{\prime}\right) e^{-i E_{21} t^{\prime}}\left[R_{1112}^{>}\left(t^{\prime}\right)-R_{1222}^{<}\left(t^{\prime}\right)\right]\right\} d t^{\prime}, \\
\dot{\rho}_{12}(t)=i E_{21} \rho_{12}(t)+\int_{0}^{t}\left\{\left[\rho_{21}\left(t-t^{\prime}\right)-\rho_{12}\left(t-t^{\prime}\right)\right]\left[R_{1221}^{<}\left(t^{\prime}\right)+R_{1221}^{>}\left(t^{\prime}\right)\right]\right. \\
\left.+\rho_{12}\left(t-t^{\prime}\right) e^{i E_{21} t^{\prime}}\left[R_{1122}^{>}\left(t^{\prime}\right)-R_{1111}^{>}\left(t^{\prime}\right)+R_{1122}^{<}\left(t^{\prime}\right)-R_{2222}^{<}\left(t^{\prime}\right)\right]\right\} d t^{\prime} .
\end{array}
$$

[The equations for $\rho_{21}(t)$ and $\rho_{22}(t)$ follow from the relations $\rho_{21}(t)=\rho_{12}^{*}(t)$ and $\rho_{22}(t)=1-\rho_{11}(t)$.] Here, $E_{21}=E_{2}-E_{1}$ is the energy difference between the bonding and antibonding orbitals, and

$R_{i j k l}^{<,>}(t)=\int d \mathbf{r} d \mathbf{r}^{\prime} d \mathbf{s} d \mathbf{s}^{\prime} \frac{\varphi_{i}(\mathbf{r}) \varphi_{j}(\mathbf{r})}{\epsilon_{r}\left|\mathbf{r}-\mathbf{r}^{\prime}\right|} \frac{\varphi_{k}(\mathbf{s}) \varphi_{l}(\mathbf{s})}{\epsilon_{r}\left|\mathbf{s}-\mathbf{s}^{\prime}\right|} \mathcal{G}^{<,>}\left(\mathbf{r}^{\prime}, \mathbf{s}^{\prime}, t\right)$,

in which the density-density correlation functions of the gate appear:

$$
\begin{aligned}
\mathcal{G}^{>} & (\mathbf{r}, \mathbf{s}, t) \\
& =\operatorname{Tr}_{B}\left(e^{-\beta\left(H_{B}-\Omega\right)} e^{i H_{B} t} \Psi^{\dagger}(\mathbf{r}) \Psi(\mathbf{r}) e^{-i H_{B} t} \Psi^{\dagger}(\mathbf{s}) \Psi(\mathbf{s})\right) \\
& =<n(\mathbf{r}, t) n(\mathbf{s})> \\
\mathcal{G}^{<} & (\mathbf{r}, \mathbf{s}, t) \\
& =\operatorname{Tr}_{B}\left(e^{-\beta\left(H_{B}-\Omega\right)} \Psi^{\dagger}(\mathbf{s}) \Psi(\mathbf{s}) e^{i H_{B} t} \Psi^{\dagger}(\mathbf{r}) \Psi(\mathbf{r}) e^{-i H_{B} t}\right) \\
& =<n(\mathbf{s}) n(\mathbf{r}, t)>.
\end{aligned}
$$

Here, $\langle X>$ denotes the thermal expectation value of the operator $X$, and we follow the notation of Kadanoff and Baym [24] in which the "greater-than" function has the order of its arguments $(\mathbf{r}, \mathbf{s})$ preserved, while the "lessthan" function has the order reversed. (Strictly speaking, the "less-than" correlation function of an operator $X(\mathbf{r}, t)$ is defined by $\mathcal{G}_{X}^{<}\left(\mathbf{r} t, \mathbf{r}^{\prime} t^{\prime}\right) \equiv<X^{\dagger}\left(\mathbf{r}^{\prime}, t^{\prime}\right) X(\mathbf{r}, t)>$; in writing Eqs. (5a)-(5b), we have used invariance in time to eliminate one of the time variables, and also noted that the density operator, $n(\mathbf{r}, t)=n^{\dagger}(\mathbf{r}, t)$, is Hermitian.)

Although they appear complicated, the various components of Eqs. (3a)-(3b) admit a simple physical interpretation. The first line of Eq. (3a) is similar to a typical rate equation, in which the rate of change of a quantity is given by the difference between the "scattering in" and the "scattering out" rates. These scattering rates are determined by the off-diagonal elements of the interation Hamiltonian, which give rise to $R_{1221}^{<,>}(t)$. The oscillatory factors are related to energy conservation. The second line of Eq. (3a) is not relevant to the current work, as it vanishes due to symmetry [see the discussion following Eq. (11)]. Eq. (3b) describes the evolution of $\rho_{12}$ and determines the dephasing of the system. The first line consists of the energy-mismatch term (which causes $\rho_{12}$ to oscillate), as well as the dephasing due to scattering, which is apparent from its dependence on $R_{1221}^{<,>}(t)$. The second line, which is related to the diagonal elements of the interaction Hamiltonian, arises as the metal gate measures the state of the quantum dot without scattering the electron. This is referred to as "pure dephasing".

\section{B. Response function of the gate electrode}

We now relate the functions $\mathcal{G}^{<,>}(\mathbf{r}, \mathbf{s}, t)$ to known properties of the metal gate. Note that $\mathcal{G}^{<,>}$describe density-density correlations of the gate. To obtain $\mathcal{G}^{<,>}(t)$, we make use of the generalized reflection coefficient $g(q, \omega)$, which is a well known quantity in surface science $[25,26]$. The function $g(q, \omega)$ is defined as the coefficient of the reflected potential induced by a point charge near a surface. More precisely, given a potential

$$
\phi_{\text {ext }}(\mathbf{r}, t)=\frac{2 \pi}{q} e^{-q(z+d)} e^{i \mathbf{q}_{\|} \cdot \mathbf{r}_{\|}} e^{i \omega t},
$$

the induced potential due to the image charge will be

$$
\phi_{\text {ind }}(\mathbf{r}, t)=-g(q, \omega) \frac{2 \pi}{q} e^{q(z-d)} e^{i \mathbf{q}_{\|} \cdot \mathbf{r}_{\|}} e^{i \omega t} .
$$

If the dot is reasonably far away from the gate (farther than about 10nm [26]), then the approximate form for $g$ is

$$
g(q, \omega)=\frac{1}{\epsilon_{r}} \frac{\epsilon(\omega)-1}{\epsilon(\omega)+1} .
$$

(Linear- and higher-order corrections in $q$ are only relevant when the dot is so close to the gate that the electric field lines are not orthogonal to the surface $[25,26]$.)

An alternative method of evaluating $g(q, \omega)$ is to determine the linear response of the surface charge density to a potential of the form (6). The induced charge density is related by a Kubo formula to the retarded densitydensity correlation function [27]

$$
\mathcal{G}_{\text {ret }}(\mathbf{r}, \mathbf{s}, t) \equiv i \theta(t)\left[\mathcal{G}^{>}(\mathbf{r}, \mathbf{s}, t)-\mathcal{G}^{<}(\mathbf{r}, \mathbf{s}, t)\right] .
$$

By the Kubo formula, we may relate $g(q, \omega)$ to the correlation functions $\mathcal{G}^{<,>}(\mathbf{r}, \mathbf{s}, t)$. The details of this calculation are straightforward, and we find that

$$
\mathcal{G}^{>}(\mathbf{q}, \omega)=e^{\beta \omega} \mathcal{G}^{<}(\mathbf{q}, \omega)=\frac{2 q \epsilon_{r}}{(2 \pi)^{5}\left(1-e^{-\beta \omega}\right)} \operatorname{Im}[g(q, \omega)]
$$


where $\mathcal{G}^{<,>}(\mathbf{q}, \omega)$ is the Fourier transform of $\mathcal{G}^{<,>}(\mathbf{r}, \mathbf{s}, t)$ (and we have used the translational invariance of the surface to write $\mathcal{G}^{<,>}(\mathbf{q}, \omega)$ as functions of a single momentum). Using Eqs. (8) and (9) in Eq. (4), we find $R_{i j k l}^{>}(t)=R_{i j k l}^{<}(-t)$ and

$$
\begin{aligned}
R_{i j k l}^{>}(t) & =\frac{1}{2 \pi^{2} \epsilon_{r}^{2}} \int d \omega \frac{e^{-i \omega t}}{1-e^{-\beta \omega}} \operatorname{Im}\left[\frac{\epsilon(\omega)-1}{\epsilon(\omega)+1}\right] \\
& \times \int d \mathbf{q}_{\|} \frac{e^{-2\left|\mathbf{q}_{\|}\right| d}}{\left|\mathbf{q}_{\|}\right|} \alpha_{i j}\left(-\mathbf{q}_{\|}\right) \alpha_{k l}\left(\mathbf{q}_{\|}\right),
\end{aligned}
$$

where

$$
\alpha_{i j}\left(\mathbf{q}_{\|}\right)=\int_{z<d} d \mathbf{r} \varphi_{i}(\mathbf{r}) \varphi_{j}(\mathbf{r}) e^{\left|\mathbf{q}_{\|}\right| z} e^{i \mathbf{q}_{\|} \cdot \mathbf{r}_{\|}}
$$

Using the symmetry properties of the qubit orbitals (the bonding and antibonding orbitals are respectively even and odd in $x$ ) in Eq. (10), we see that

$$
R_{1112}^{<,>}=0=R_{1222}^{<,>} .
$$

Thus the second line of Eq. (3a) vanishes, and Eqs. (3a) and (3b) may be solved separately.
We see from Eq. (10) that $R_{i j k l}^{<,>}(t)$ is a product of an orbital term and a time-dependent term:

$$
R_{i j k l}^{<,>}(t)=I^{<,>}(t) \Gamma_{i j k l},
$$

where

$$
\Gamma_{i j k l}=\frac{1}{2 \pi^{2} \epsilon_{r}^{2}} \int d \mathbf{q}_{\|} \frac{e^{-2\left|\mathbf{q}_{\|}\right| d}}{\left|\mathbf{q}_{\|}\right|} \alpha_{i j}\left(-\mathbf{q}_{\|}\right) \alpha_{k l}\left(\mathbf{q}_{\|}\right)
$$

and

$$
I^{>}(t)=I^{<}(-t)=\int d \omega \frac{e^{-i \omega t}}{1-e^{-\beta \omega}} \operatorname{Im}\left[\frac{\epsilon(\omega)-1}{\epsilon(\omega)+1}\right]
$$

Assuming the Drude form [22] for $\epsilon(\omega)$,

$$
\epsilon(\omega)=1-\frac{\omega_{p}^{2}}{\omega(\omega+2 i \gamma)}
$$

$I^{>}(t)$ may be evaluated explicitly, yielding

$$
I^{>}(t) \approx \begin{cases}\frac{\pi \omega_{p}^{2} e^{-\gamma t}}{4 x} e^{-i x t}-\frac{2 \pi}{\beta} \sum_{n=1}^{\infty} e^{-2 \pi n t / \beta} g_{n} & t>0 \\ \frac{\pi \omega_{p}^{2} e^{\gamma t}}{4 x} e^{-i x t}-\frac{2 \pi}{\beta} \sum_{n=1}^{\infty} e^{2 \pi n t / \beta} g_{n} & t<0\end{cases}
$$

Here, the function $g_{n}$ is defined as

$$
g_{n}=\frac{8 \pi n \beta^{3} \gamma \omega_{p}^{2}}{4(2 \pi)^{4} n^{4}+4(2 \pi)^{2} n^{2} \beta^{2}\left(\omega_{p}^{2}-4 \gamma^{2}\right)+\omega_{p}^{4} \beta^{4}}
$$

and $x=\left(\omega_{p}^{2} / 2-\gamma^{2}\right)^{1 / 2}$ is the real part of the firstquadrant pole of $\operatorname{Im}[(\epsilon-1) /(\epsilon+1)]$. Since $\omega_{p}$ is typically of the order of $10 \mathrm{eV}, \exp (-i x t)$ oscillates extremely rapidly. In Eq. (15), terms which are a factor of $\exp \left(-\beta \omega_{p}\right)$ smaller have been neglected; for the realistic value of $\omega_{p}=10 \mathrm{eV}$, this is a reasonable approximation for temperatures as high as $1000 \mathrm{~K}$ and is certainly justified for the temperatures considered here.

\section{The Laplace transform of the kinetic equation}

We proceed now to the solution of Eqs. (3a)-(3b). Since these equations are of Volterra type, they may be solved by direct time evolution from a given initial condition. However, this method is computationally intense - to resolve the rapid oscillations of the integral kernel (15), we require a timestep $\Delta t$ of the order of $0.01 \mathrm{fs}$, meaning that $10^{8}$ timesteps are needed for a simulation time of 1 ns. Furthermore, the method scales badly with the number of timesteps - each new timestep requires an integration over all previous times. Although this may be improved somewhat (e.g. using the fact that the kernel (15) decays rapidly), simulations beyond several nanoseconds cannot be computed in reasonable time.

It is better to take advantage of the fact that Eqs. (3a)(3b) are linear integral equations with convolutive kernels, and to use the Laplace transform. We multiply Eqs. (3a)-(3b) by $\exp (-z t)$ and integrate over $t$ :

$$
z \tilde{\rho}_{11}(z)=\rho_{11}(0)+\Gamma_{1221}\left\{\tilde{\rho}_{22}(z)\left[\tilde{I}^{>}\left(z-i E_{21}\right)+\tilde{I}^{<}\left(z+i E_{21}\right)\right]-\tilde{\rho}_{11}(z)\left[\tilde{I}^{>}\left(z+i E_{21}\right)+\tilde{I}^{<}\left(z-i E_{21}\right)\right]\right\}
$$




$$
\begin{aligned}
z \tilde{\rho}_{12}(z) & =\rho_{12}(0)+i E_{21} \tilde{\rho}_{12}(z)+\Gamma_{1221}\left[\tilde{\rho}_{21}(z)-\tilde{\rho}_{12}(z)\right]\left[\tilde{I}^{>}(z)+\tilde{I}^{<}(z)\right] \\
& +\tilde{\rho}_{12}(z)\left[\left(\Gamma_{1122}-\Gamma_{1111}\right) \tilde{I}^{>}\left(z-i E_{21}\right)+\left(\Gamma_{1122}-\Gamma_{2222}\right) \tilde{I}^{<}\left(z-i E_{21}\right)\right] .
\end{aligned}
$$

Here, we have introduced the Laplace transforms of $\rho_{i j}$ and $I(t)$ :

$$
\tilde{\rho}_{i j}(z)=\int_{0}^{\infty} \rho_{i j}(t) e^{-z t} d t
$$

and

$$
\tilde{I}^{<,>}(z)=\int_{0}^{\infty} I^{<,>}(t) e^{-z t} d t
$$

Since $I^{<,>}(t)$ are sums of exponentials [see Eq. (15)], their Laplace transforms are straightforward to calculate.

Eqs. (17a)-(17b), when considered with similar equations for $\rho_{22}$ and $\rho_{21}$, form a pair of $2 \times 2$ matrix equations for the unknowns $\tilde{\rho}_{i j}(z)$. The formal solution of these is easily found, although the solutions are complicated functions of $z$, for which the inverse Laplace transform cannot be calculated. Direct computation of the inverse Laplace transform by numerical contour integration is possible; however, this method requires a separate contour integral for each different time at which the solution is desired and is of similar computational intensity to the direct simulation of Eqs. (3a)-(3b).

The alternative method is to make use of the fact that the positions of the simple poles of the Laplace transform yield the exponential decay rates. For asymptotic analysis, only the poles with the largest (and sometimes second largest) real parts are significant; the smaller the real part, the faster the decay of that component. Algebraic decay leads to singularities at the origin. Therefore, our strategy for analyzing Eqs. (17a)-(17b) is to find the poles of $\tilde{\rho}_{i j}(z)$ with the largest real parts, and to investigate the nature (multiplicity, residues, etc.) of these singularities.

\section{NUMERICAL RESULTS FOR A REALISTIC SYSTEM}

In order to obtain numerical results, we must specify the physical constants that enter Eqs. (17a)-(17b). The semiconductor surrounding the quantum dot is assumed to be GaAs/AlGaAs, for which $\epsilon_{r} \approx 13$ and $m^{*} \approx 0.067$. To describe the metal, we take the "typical" values $\omega_{p}=10 \mathrm{eV}$ and $\gamma=1 \mathrm{eV}$. The size of the quantum dots were chosen to be $R=50 \mathrm{~nm}$. The other four parameters - temperature, tunneling energy, distance between the dots and distance to the gate - were varied. When each of these was varied, the others were chosen at the following default values: temperature $T=100 \mathrm{mK}$; tunneling energy $E_{21}=8 \mu \mathrm{eV}$; distance between the dots $s=100 \mathrm{~nm}$; distance to the gate $d=50 \mathrm{~nm}$. These parameters were chosen so that our results could be compared with the experimental results of Ref. [18].

For all choices of the parameters, we found qualitatively similar results, which we now summarize. The evolution of the diagonal elements $\rho_{11}$ and $\rho_{22}$ is dominated by two real, simple poles. A pole at $z=0$ ensures that there is a nondecaying component, and another pole at $z<0$ describes the decay. From the residues at these poles, we find that the diagonal matrix elements evolve (approximately) according to

$$
\left(\begin{array}{c}
\rho_{11}(t) \\
\rho_{22}(t)
\end{array}\right)=\left(\begin{array}{rr}
a+(1-a) e^{-t / T_{1}} & a\left(1-e^{-t / T_{1}}\right) \\
(1-a)\left(1-e^{-t / T_{1}}\right) & 1-a\left(1-e^{-t / T_{1}}\right)
\end{array}\right)\left(\begin{array}{c}
\rho_{11}^{(0)} \\
\rho_{22}^{(0)}
\end{array}\right)
$$

where $a$ and $T_{1}$ are functions of the parameters. (We use the standard notation $T_{1}$ to denote the energy relaxation time.) Eq. (18) preserves the relation $\rho_{11}+\rho_{22}=1$ and shows that all initial conditions decay to the same final state.

The dynamics of the off-diagonal elements $\rho_{12}$ and $\rho_{21}$ are governed by a complex conjugate pair of simple poles, with negative real part. Most of the residues at these poles vanish, and the evolution is approximately described by

$$
\left(\begin{array}{l}
\rho_{12}(t) \\
\rho_{21}(t)
\end{array}\right)=\left(\begin{array}{lr}
e^{-t / T_{2}+i \epsilon_{21} t} & 0 \\
0 & e^{-t / T_{2}-i \epsilon_{21} t}
\end{array}\right)\left(\begin{array}{l}
\rho_{12}^{(0)} \\
\rho_{21}^{(0)}
\end{array}\right)
$$

Clearly, this evolution preserves the relation $\rho_{12}=\rho_{21}^{*}$. The oscillation frequency $\epsilon_{21}$ is always slightly larger (around 1\%) than the bare tunneling energy $E_{21}$, i.e. the gate assists the tunneling.

For all values of the parameters, we find that the dephasing rate $1 / T_{2}$ is slightly faster than half of the energy decay rate:

$$
\frac{1}{T_{2}}=\frac{1}{2 T_{1}}+\frac{1}{T_{\Phi}}
$$

where $1 / T_{\Phi}>0$ is the "pure dephasing" rate. The pure dephasing rate is always less than $1 \%$ of the value of the energy dissipation rate, which is unusual for solid state systems. However, this may be understood by considering the source of pure dephasing. As discussed at the end of Sec. III A, pure dephasing is described by the second line of Eq. (3b). (This was verified by removing this line from our numerical work - in this case, the relation $1 / T_{2}=1 / 2 T_{1}$ holds exactly.) The terms in this line are proportional to either $\left(\Gamma_{1122}-\Gamma_{1111}\right)$ or $\left(\Gamma_{1122}-\Gamma_{2222}\right)$ which, from Eq. (13), are related to the differences between the multipole moments of the bonding and antibonding orbitals. These moments are very similar, so the 

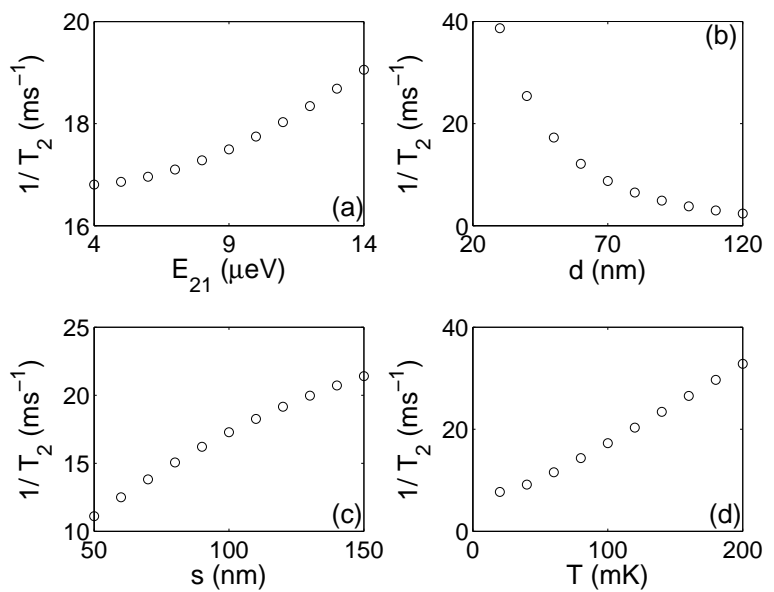

FIG. 2: The dephasing rate of the double quantum dot system, due to the interaction with the gate electrode, as a function of tunneling energy (a), distance between the gate and the quantum dots (b), distance between the quantum dots (c) and temperature (d). In (a) we set $d=50 \mathrm{~nm}, s=100 \mathrm{~nm}$ and $T=100 \mathrm{mK}$. In (b) $E_{21}=8 \mu \mathrm{eV}, s=100 \mathrm{~nm}$ and $T=100 \mathrm{mK}$. In (c) $E_{21}=8 \mu \mathrm{eV}, d=50 \mathrm{~nm}$ and $T=100 \mathrm{mK}$, and finally in (d) we used $E_{21}=8 \mu \mathrm{eV}, d=50 \mathrm{~nm}$ and $s=100 \mathrm{~nm}$.

pure dephasing rate may be expected to be small. The physical interpretation is that the gate cannot perform an accurate quantum measurement, since it cannot readily distinguish between the quantum states.

Our numerical results are contained in Figs. 2-4. Fig. 2 shows the dephasing rate as a function of $E_{21}, d, s$ and $T$. The dephasing rate clearly increases very rapidly as $d$ is reduced, or as the tunneling energy is increased. Comparing Fig. 2(a) with Fig. 3(b) of Ref. [18], we see that the dependence of dephasing on tunneling energy is far stronger for the Coulomb interaction than for the electron-phonon interaction. Thus for very large tunneling energies, we expect competition between these effects.

The "pure dephasing" rate is plotted in Fig. 3, and is clearly far smaller than the total dephasing rate. Finally, in Fig. 4 we show the asymptotic value of $\rho_{11}$, compared with the thermal equilibrium value $1 /\left(1+e^{-\beta E_{21}}\right)$. It is clear from the agreement between these two quantities that the final qubit temperature is the same as that of the gate. We note that there is negligible difference between using $E_{21}$ (the bare tunneling energy) and $\epsilon_{21}$ (the true oscillation frequency) to evaluate the thermal equilibrium distribution. The results using $E_{21}$ were consistently closer to the numerically determined asymptotics, but both results were always within $0.1 \%$ of each other.

\section{CONCLUSIONS AND OUTLOOK}

We have studied dephasing of a charge quantum dot due to the direct Coulomb interaction with nearby gate electrodes. The interaction was treated in the second order Born approximation, using the kinetic equation
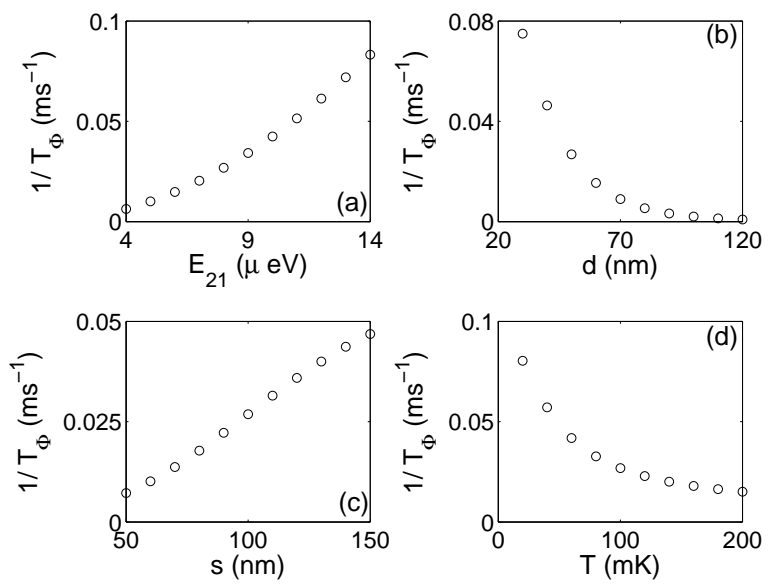

FIG. 3: The pure dephasing rate, as defined in Eq. (20), as a function of tunneling energy (a), distance between the gate and the quantum dots (b), distance between the quantum dots (c) and temperature $(\mathrm{d})$. The other parameters are chosen as in Figs. 2(a)-(d), respectively.

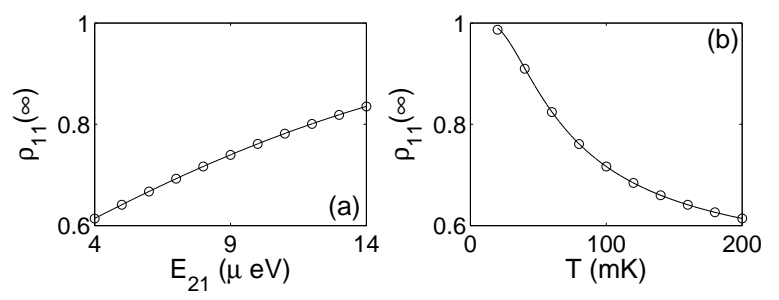

FIG. 4: The asymptotic value of $\rho_{11}$, as a function of $E_{21}$ (with $T=100 \mathrm{mK}$ ) and $T$ (with $E_{21}=8 \mu \mathrm{eV}$ ). The solid line indicates the thermal equilibrium value, while the circles are the numerically calculated asymptotes (calculated from the residue of the $z=0$ pole of the Laplace transform). In both figures, $s=100 \mathrm{~nm}$ and $d=50 \mathrm{~nm}$.

method [20]. Both the dephasing and dissipation times have been evaluated by numerical solution of Laplace transform of the kinetic equation.

The dephasing and dissipation rates were found to be of the order of $10 \mathrm{~ms}^{-1}$. This is significantly slower than the typical phonon dephasing rate $[17,18]$, which is usually around $1 \mathrm{~ns}^{-1}$ for the system parameters considered here. Gate-induced dephasing yields a negligible contribution for the current design of double quantum dots. However, the dephasing rate increases rapidly as either the system becomes smaller or the tunneling energy is increased. Therefore as systems are miniaturized (by decreasing $d$ ) or higher oscillation frequencies are sought (by increasing $E_{21}$ ), this form of dephasing will become increasingly relevant.

\section{Acknowledgements}

We thank A. Liebsch and R. O. Jones for many stimulating discussions and helpful insights. 


\section{Appendix A: Derivation of the kinetic equation}

We derive Eqs. (3a)-(3b), the kinetic equations describing the evolution of the quantum dot under the influence of the gate electrode, following closely the method of Haake [20].

The Hamiltonian describing the combined dot-gate system is

$$
\begin{aligned}
& H=\sum_{i=1}^{2} E_{i} a_{i}^{\dagger} a_{i}+H_{B} \\
& +\sum_{i, j} \int d \mathbf{r} d \mathbf{r}^{\prime} \frac{1}{\epsilon_{r}\left|\mathbf{r}-\mathbf{r}^{\prime}\right|} \varphi_{i}^{*}(\mathbf{r}) \varphi_{j}(\mathbf{r}) \Psi^{\dagger}\left(\mathbf{r}^{\prime}\right) \Psi\left(\mathbf{r}^{\prime}\right) a_{i}^{\dagger} a_{j} .(\mathrm{A} 1)
\end{aligned}
$$

The reduced density matrix of the dot is defined as

$$
\rho(t)=\operatorname{Tr}_{B} W(t),
$$

where $W(t)$ is the density matrix for the full system, and $\operatorname{Tr}_{B}$ is the partial trace over the gate degrees of freedom. We assume that at $t=0$, the gate and the dot system are disentangled, and that the gate is in a thermal distribution:

$$
W(0)=\rho_{0} \otimes e^{-\beta\left(H_{B}-\Omega\right)}
$$

with $e^{-\beta \Omega}=\operatorname{Tr}_{B}\left(e^{-\beta H_{B}}\right)$.

The density operator $W(t)$ evolves according to the Heisenberg equation of motion,

$$
\dot{W}(t)=-i[H, W(t)] \equiv-i L W(t),
$$

where $L$ is the Liouvillian superoperator. We apply the projection superoperators $\mathcal{P}=e^{-\beta\left(H_{B}-\Omega\right)} \operatorname{Tr}_{B}$ and $\mathcal{P}^{\prime}=$ $1-\mathcal{P}$ to this equation, integrate the equation for $\mathcal{P}^{\prime} W(t)$, and perform the partial trace over $\mathcal{P} W(t)$ to obtain (see Ref. [20] for details)

$$
\dot{\rho}(t)=-i L_{e f f} \rho(t)+\int_{0}^{t} K\left(t^{\prime}\right) \rho\left(t-t^{\prime}\right) d t^{\prime},
$$

where

$$
L_{e f f}=L_{S}+\operatorname{Tr}_{B} L_{S B} e^{-\beta\left(H_{B}-\Omega\right)}
$$

and

$$
\begin{aligned}
& K(t)=-\operatorname{Tr}_{B}\left\{L_{S B} \exp [-i(1-\mathcal{P}) L t]\right. \\
& \left.\quad \times(1-\mathcal{P})\left(L_{B}+L_{S B}\right) e^{-\beta\left(H_{B}-\Omega\right)}\right\} .
\end{aligned}
$$

The superoperators $L_{B}, L_{S}$ and $L_{S B}$ are the Liouvillians of the gate, the dot and the interaction term respectively. [Eqs. (A4)-(A6) are Eqs. (2b.13) and (2b.14) in Ref. [20]; the term $I(t)$ found in Ref. [20] vanishes due to our choice of projector.] Since $L_{B} H_{B} \equiv 0, K(t)$ contains only terms of second order and higher in the interaction term.

Eq. (A4) is formally exact, but through the term $\exp [-i(1-\mathcal{P}) L t]$ in Eq. $(\mathrm{A} 6), K(t)$ contains correlation functions of all orders. Assuming that the lowest order correlators are the most important, we write

$$
\exp [-i(1-\mathcal{P}) L t] \approx \exp \left[-i(1-\mathcal{P})\left(L_{S}+L_{B}\right) t\right] .
$$

Then Eq. (A6) retains only terms of second order in the interaction.

We note that this approximation contains all secondorder processes. This may be checked by writing

$$
\rho_{i j}(t)=\operatorname{Tr}\left[a_{j}^{\dagger}(t) a_{i}(t) \rho_{0} \otimes e^{-\beta\left(H_{B}-\Omega\right)}\right]
$$

and expanding $\rho_{i j}(t)$ to second order. We thus find that Eq. (A4) is the time representation of the Dyson equation for $\rho_{i j}(t)$ in the second order Born approximation.

By substituting the Hamiltonian (A1) into Eq. (A4), using the approximation (A7) and performing the requisite commutators and traces, we obtain the kinetic equations (3a) and (3b) studied in the text. Furthermore, if we use Eq. (A4) to derive equations for $\dot{\rho}_{21}$ and $\dot{\rho}_{22}$ in the second order approximation, we find that they exactly obey the symmetries

$$
\dot{\rho}_{11}+\dot{\rho}_{22}=0 \text { and } \quad \dot{\rho}_{12}^{*}=\dot{\rho}_{21} .
$$

Thus our approximation scheme is consistent - it conserves both the hermiticity of $\rho(t)$ and the property that $\operatorname{Tr}[\rho(t)]=1$.

\section{Appendix B: Numerical aspects}

\section{The qubit wavefunctions}

To calculate the $\Gamma_{i j k l}$ components needed in Eqs. (17a)-(17b), the wavefunctions of the quantum dot are needed. These are obtained by approximating the $z$-dependence with a $\delta$-function [see Eq. (1)] and solving numerically the 2-dimensional Schrödinger equation

$$
\left(-\frac{1}{2 m^{*}} \nabla_{\|}^{2}+V\left(\mathbf{r}_{\|}\right)\right) \varphi_{i}\left(\mathbf{r}_{\|}\right)=E_{i} \varphi_{i}\left(\mathbf{r}_{\|}\right)
$$

with the potential

$$
V\left(\mathbf{r}_{\|}\right)=\left\{\begin{array}{l}
0 \quad x \in\left[-R-\frac{s}{2},-\frac{s}{2}\right] ; \quad y \in\left[-\frac{R}{2}, \frac{R}{2}\right] \\
V_{0} \quad x \in\left[-\frac{s}{2}, \frac{s}{2}\right] ; \quad y \in\left[-\frac{R}{2}, \frac{R}{2}\right] \\
0 \quad x \in\left[\frac{s}{2}, R+\frac{s}{2}\right] ; \quad y \in\left[-\frac{R}{2}, \frac{R}{2}\right] \\
\infty \quad \text { otherwise }
\end{array}\right.
$$

Here, $V_{0}$ is chosen to reproduce a given tunneling energy, $E_{21}$.

Eq. (B1) is separable. The $y$ component is calculated analytically, and the $x$ component is determined numerically by expanding $\varphi$ in a cosine or sine series (for the bonding and antibonding orbitals respectively); we used 500 modes in this series (which yielded the same result as using 300 modes). Using the expansion in Fourier modes, the integral in Eq. (11) may be obtained analytically. The integral in Eq. (13) must be evaluated numerically, with some care taken to resolve the apparent singularity at the origin. 


\section{Poles of the Laplace transform}

Eqs. (17a)-(17b), when complemented with the equations for $\tilde{\rho}_{21}$ and $\tilde{\rho}_{22}$, form a pair of matrix equations [that is, one equation for $\left(\rho_{11}, \rho_{22}\right)$ and one for $\left.\left(\rho_{12}, \rho_{21}\right)\right]$, each of the form

$$
\tilde{U}(z) \tilde{\mathbf{f}}(z)=\mathbf{f}_{0}
$$

The poles of $\tilde{\mathbf{f}}(z)$ occur at the values of $z$ for which the determinant of $\tilde{U}(z)$ vanishes. Thus we are left with a simple root-finding problem, which we solve using the Newton-Raphson method. Then using Cramer's rule (which is particularly simple in this case, since $\tilde{U}(z)$ is a $2 \times 2$ matrix), we may explicitly solve Eq. (B3), and calculate the residues of the components of $\tilde{U}^{-1}(z)$ at the poles using finite differences.

The bulk of the computation time is occupied by the Newton-Raphson calculation, due to the infinite series in $\tilde{I}^{>}(z)$. From Eq. (15), we find

$$
\tilde{I}^{>}(z) \approx \frac{\pi \omega_{p}^{2}}{4 x(z+\gamma+i x)}-\frac{2 \pi}{\beta} \sum_{n=1}^{\infty} \frac{g_{n}}{z+2 \pi n / \beta},
$$

with $g_{n}$ defined in Eq. (16). This series may be approximated analytically in the limits $|z| \beta \gg 1$ and $|z| \beta \ll 1$; however, when solving Eqs. (17a)-(17b) we must evaluate $\tilde{I}^{>}(z)$ with $|z| \approx E_{21}$, which is of similar magnitude to $1 / \beta$. Thus we cannot use an analytic approximation for the series in Eq. (B4); it must be truncated at some value $N$ and calculated numerically.

The factor $g_{n}$ decays as $n^{-3}$ provided $n \gg \beta \omega_{p} \equiv$ $\omega_{p} / k_{B} T$. Since the plasmon energy is typically much larger than the temperature, the series needs very large $N$. However, if $N$ is too large, the accuracy may be reduced due to round-off errors. This problem is aggravated by the almost complete cancellation of the first term of Eq. (B4) with the series - typically, the series sums to approximately 0.2 , while $\tilde{I}^{>}(z) \approx 10^{-6}-i \pi / 2$.

We found that the optimal number of terms $N \sim 10^{8}$. (For $T>40 \mathrm{mK}$, the tail of the series is negligible if $N=1 \times 10^{8}$ terms are used; for smaller temperatures, $N=4 \times 10^{8}$ terms were needed.) The maximum possible round-off error (using double-precision arithmetic) for this number of sums is approximately $4 \times 10^{-9}$, which is sufficiently smaller than $\tilde{I}^{>}(z)$ that it does not change our results. To verify that round-off effects were negligible, we reversed the order of the sum, and we also broke the term $\pi \omega_{p}^{2} / 4 x(z+\gamma+i x)$ into $N$ terms and included these terms in the summation; neither of these modifications significantly affected the result.
[1] P. W. Shor, Proceedings of the 35th Annual Symposium on the Foundations of Computer Science, IEEE Computer Society, Los Alamitos (1994)

[2] D. P. DiVincenzo, Fortschr. Phys. 48, 771 (2000)

[3] M. Nielsen and I. L. Chuang, Quantum Computation and Quantum Information, Cambridge University Press, Cambridge (2000)

[4] A. Shnirman, G. Schön and Z. Hermon, Phys. Rev. Lett. 79, 2371 (1997); Yu. Makhlin, G. Schön, and A. Shnirman, Rev. Mod. Phys. 73,357 (2001)

[5] D. Averin, Solid State Commun. 105, 659 (1998)

[6] V. Bouchiat, D. Vion, P. Joyez, D. Esteve and M. H. Devoret, Phys. Scr. T 76, 165 (1998)

[7] D. Loss and D. P. DiVincenzo, Phys. Rev. A 57, 120 (1998)

[8] M. S. Sherwin, A. Imamoglu and T. Montroy, Phys. Rev. A 60, 3508 (1999)

[9] B. E. Kane, Nature 393, 133 (1998)

[10] S. D. Barrett and G. J. Milburn, Phys. Rev. B 68, 155307 (2003)

[11] L. Jacak, J. Krasnyj, W. Jacak, R. Gonczarek and P. Machnikowski, Phys. Rev. B 72, 245309 (2005)

[12] E. Buks, R. Schuster, M. Heiblum, D. Mahalu and V. Umansky, Nature 391, 871 (1998)

[13] Y. Levinson, Europhys. Lett. 39, 299 (1997)

[14] D. Sprinzak, E. Buks, M. Heiblum and H. Shtrikman,
Phys. Rev. Lett. 84, 5820 (2000)

[15] A. Silva and S. Levit, Phys. Rev. B 63, 201309(R) (2001)

[16] A. D. Armour and M. P. Blencowe, Phys. Rev. B 64, 035311 (2001)

[17] V. N. Stavrou and Xuedong Hu, Phys. Rev. B 72, 075362 (2005)

[18] T. Hayashi, T. Fujisawa, H. D. Cheong, Y. H. Jeong and Y. Hirayama, Phys. Rev. Lett. 91, 226804 (2003)

[19] K. M. Indlekofer, cond-mat/0610806

[20] F. Haake, Quantum statistics in optics and solid state physics , Springer Tracts in Modern Physics 66, Springer, Berlin (1973)

[21] D. Loss and H. Schoeller, J. Stat. Phys. 56, 175 (1989)

[22] P. Drude, Ann. der Phys. 14, 936 (1904)

[23] A. W. Holleitner, R. H. Blick, A.K. Hüttel, K. Eberl and J.P. Kotthaus, Science 297, 70 (2002)

[24] L. P. Kadanoff and G. Baym, Quantum statistical mechanics, W. A. Benjamin Inc., New York (1962)

[25] A. Liebsch, Electronic excitations at metal surfaces, Plenum Press, New York (1997)

[26] B. N. J. Persson, Sliding friction : physical principles and applications, Springer, Berlin (2000)

[27] G. D. Mahan, Many particle physics, Plenum Press, New York (1990) 